\title{
Arteries: anatomy, imaging, clinical, and surgical relevance
}

\section{Fabrice Duparc ${ }^{1} \cdot$ Bruno Grignon ${ }^{2}$}

Published online: 2 June 2018

c) Springer-Verlag France SAS, part of Springer Nature 2018

The main part of this issue (Surg Radiol Anat 2018, 07) is devoted to the clinical anatomy of "Arteries". When searching with using the term "artery" in the titles of the submitted manuscripts, it appears that more than 100 submissions to our journal per year are related to this field of anatomical research.

The articles included in this issue show the large distribution of these studies: abdominal wall, intra-abdominal cavity, retroperitoneal space, heart and aorta, limbs, head, and neck. The intrastructural aspect of the arterial networks is also presented through the study of vasa-vasorum.
The aberrant arteries and the numerous anatomical variations of vessels represent some important risks during medical imaging and surgical procedures, and the knowledge of these unusual aspects and topographies is necessary for specialists in daily practice.

In this various and rich part of anatomical studies about the vessels, some specific indications in plastic surgery concern the perforators and perforasomes, the pedicled flaps, and the anatomical basis of techniques of rerouting arteries, and will be grouped in the future Surg Radiol Anat issue devoted to Anatomy and Plastic Surgery.

Fabrice Duparc

fabrice.duparc@univ-rouen.fr

Bruno Grignon

b.grignon@chru-nancy.fr

1 Laboratory of Anatomy, Faculty of Medicine, Rouen

University, 22 Boulevard Gambetta, 76183 Rouen, France

2 Department of Medical Imaging, Nancy University Hospital, 29 avenue du Maréchal de Lattre de Tassigny, 54035 Nancy, France 